\title{
EDM Characteristics of CVD-Carbon Electrode
}

\author{
Yoshiyuki UNO*, Akira OKADA*, Hirohito NAKANISHI*, Changning GUO*, \\ Yasuhiro OKAMOTO* and Takashi TAKAGI**
}

(Received on April 7, 1997)

\author{
* Department of Mechanical Engineering, Okayama University, Okayama 700, JAPAN \\ ** Ibiden Co., Ltd., Ogaki 503, JAPAN
}

\begin{abstract}
In EDM process, the reduction of electrode wear is very important for high accuracy machining. EDM with low electrode wear rate can be done by adhesion of heat resolved carbon to the electrode end surface. However, this phenomenon is observed only under the long pulse duration. Therefore, EDM with low electrode wear rate under finishing condition is impossible so far. In this study, EDM characteristics of CVD-carbon electrode are experimentally investigated for higher performance EDM. This electrode is made by chemical vapor deposition method and its structure is similar to the heat resolved carbon adhering to the electrode end surface. Experimental analysis made it clear that EDM with low electrode wear rate is possible by using CVD-carbon electrode even under finishing condition, since the heat resolved carbon adhered to the end surface of CVDcarbon electrode has a stronger adhering ability than that of graphite one.
\end{abstract}

Key Words : EDM, CVD-carbon, low electrode wear, finishing condition

\section{INTRODUCTION}

In electrical discharge machining (EDM) process, it is very important to reduce not only surface roughness but also electrode wear for high accuracy machining. It was reported that the EDM with low electrode wear rate could be achieved by adhesions of heat resolved carbon from machining fluid and workpiece material to the electrode end surface, because of the protection effect of the former which has large heat resistivity ${ }^{1), 2}$. Under a proper condition, even EDM without electrode wear is possible. Such adhesion phenomenon, however, is observed only under the condition that pulse duration is relatively large ${ }^{3)}$. That is, it never appears on the small pulse duration employed in the finishing condition. Therefore, EDM with low electrode wear rate under finishing condition is impossible so far and the tolerable electrode wear in the finishing EDM is practically allowed to be inevitable.

Mohri et al. ${ }^{4}$ reported that the heat resolved carbon from machining fluid adhering to the electrode end surface was composed of turbostratic carbon. It has the chemical structure in which hexagonal lattice plane laminates irregularly, different from graphite structure. From these results, it is considered that EDM with low electrode wear rate might be attained by using turbostratic carbon electrode even under the finishing condition.

In this study, EDM characteristics of CVDcarbon electrode with turbostratic structure are experimentally investigated for higher performance EDM.

\section{CVD-CARBON}

An electrode used in this study is made by chemical vapor deposition(CVD) method. When 1,2-dichloroethylene as vapor source and argon as carrier gas are mixed and sent to a graphite plate heated up to about $1500^{\circ} \mathrm{C}$ in the plating room, CVD-carbon is generated on the graphite plate surface as shown in Fig. . $^{\text {5) }}$. In laminar (a), hexagonal lattice plane laminates parallel to the surface of graphite plate but irregularly, different from graphite

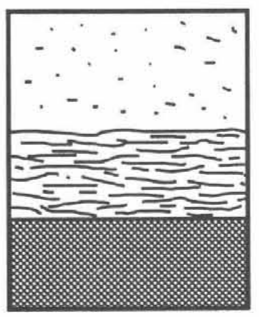

(a)Laminar

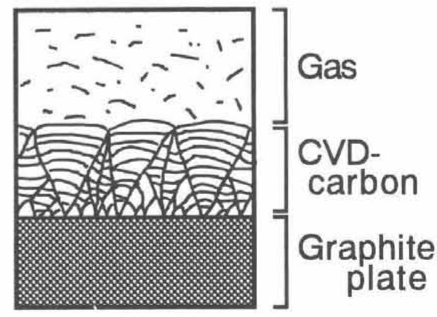

(b) Columnar
Fig.1 Structure of CVD-carbon 
structure. That is, the structure is turbostratic in this case. In columnar (b), arcuate lattice plane laminates around the carbon which is previously resolved in the vapor and adhered to the graphite plate. The structure of CVD-carbon used in this study as an electrode is a mixture of laminar and columnar. It is very similar to the heat resolved carbon made from kerosine type machining fluid adhering to the electrode end surface in EDM process.

Table 1 shows the physical properties of CVDcarbon. As shown in the table, resistivity and heat conductivity in the layer direction greatly differ from those in the thickness direction, since the structure of CVD-carbon is accumulated layer by layer. That is, in the layer direction, an electric current flows easily and heat also conducts quickly. In other words, the structure of CVD-carbon is highly anisotropic. It is also shown that the specific gravity of CVD-carbon is larger than that of graphite. It means that the structure of CVD-carbon is denser than that of graphite.

\section{EXPERIMENTAL PROCEDURES}

Experiments are performed using the NC electrical discharge machine with transistor switching circuit. Machining conditions are set as shown in Table 2. Carbon tool steel SK3 in JIS specification is used as a workpiece. Fig. 2 shows the shape and structure of CVD-carbon electrode. This electrode consists of bundled carbon fibers of about $1 \mathrm{~mm}$ in diameter used as base material and heat resolved carbon generated around these fibers by CVD method. The diameter of the electrode is $6 \mathrm{~mm}$. As shown in the figure, the resistivity of radial direction $\rho$ is $50000 \mu \Omega \cdot \mathrm{cm}$ and the heat conductivity $\lambda$ is $2 \mathrm{~W} /(\mathrm{m} \cdot \mathrm{K})$, while those of axis direction are $700 \mu$ $\Omega \cdot \mathrm{cm}$ and $300 \mathrm{~W} /(\mathrm{m} \cdot \mathrm{K})$ respectively, since CVDcarbon is deposited radially. Graphite electrode of the same shape is also used for comparison.

\section{RESULTS AND DISCUSSION}

\subsection{EDM characteristics}

Fig. 3 shows the relationship between the electrode wear and the pulse duration. The electrode wear was calculated by the change of its length. As can be seen from the figure, the electrode wear decreases with an increase of the pulse duration in
Table 1 Physical properties of CVD-carbon and graphite

\begin{tabular}{|c|c|r|c|}
\hline & $\begin{array}{c}\text { Specific } \\
\text { gravity } \\
\mathrm{g} / \mathrm{cm}^{2}\end{array}$ & $\begin{array}{r}\text { Resistivity } \\
\mu \cdot \mathrm{cm}\end{array}$ & $\begin{array}{l}\text { Heat } \\
\text { conductivity } \\
\mathrm{W} /(\mathrm{m} \cdot \mathrm{K})\end{array}$ \\
\hline CVD-carbon & 2.0 & $\begin{array}{l}(\perp) 50000 \\
(/ /) 700\end{array}$ & 2 \\
\hline Graphite & 1.8 & 1400 & 75.4 \\
\hline
\end{tabular}

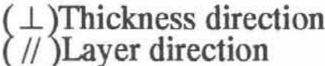

Table 2 Machining conditions

\begin{tabular}{|l|l|}
\hline Polarity & Electrode $:(+)$ \\
\hline Non-loaded voltage & E0 $=120 \mathrm{~V}$ \\
\hline Discharge current & $\mathrm{I} p=5 \mathrm{~A}$ \\
\hline Pulse duration & $\tau \mathrm{p}=4 \sim 100 \mu \mathrm{s}$ \\
\hline Duty factor & D.F. $=50 \%$ \\
\hline Machining fluid & Kerosine type fluid \\
\hline
\end{tabular}

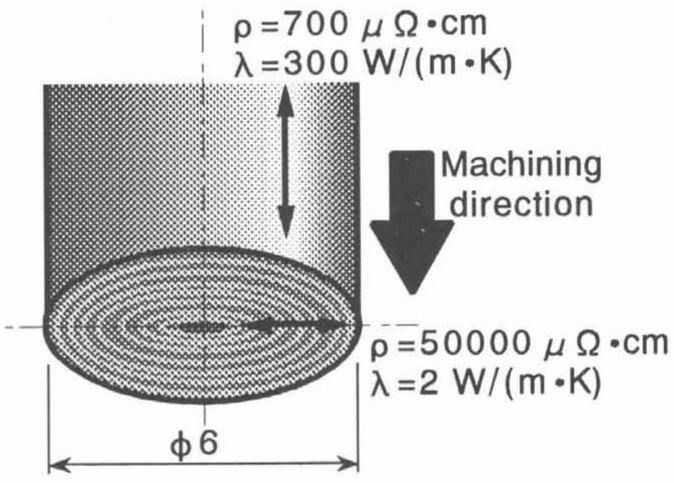

Fig.2 Shape and structure of CVD-carbon electrode

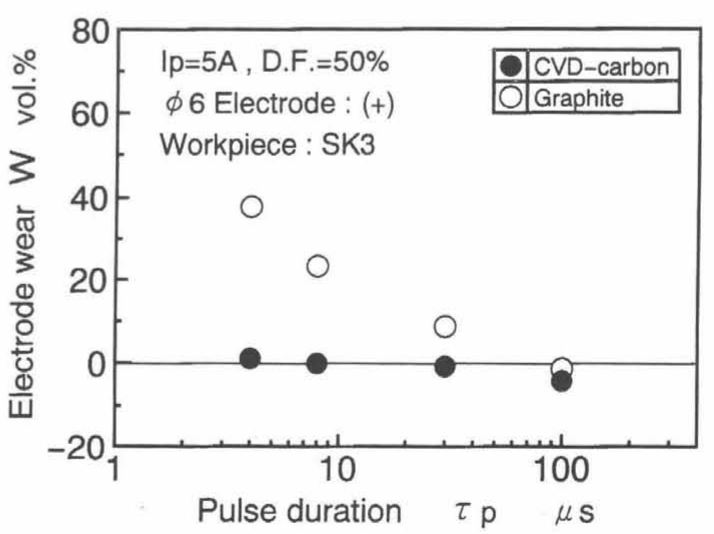

Fig.3 Relationship between electrode wear and pulse duration 


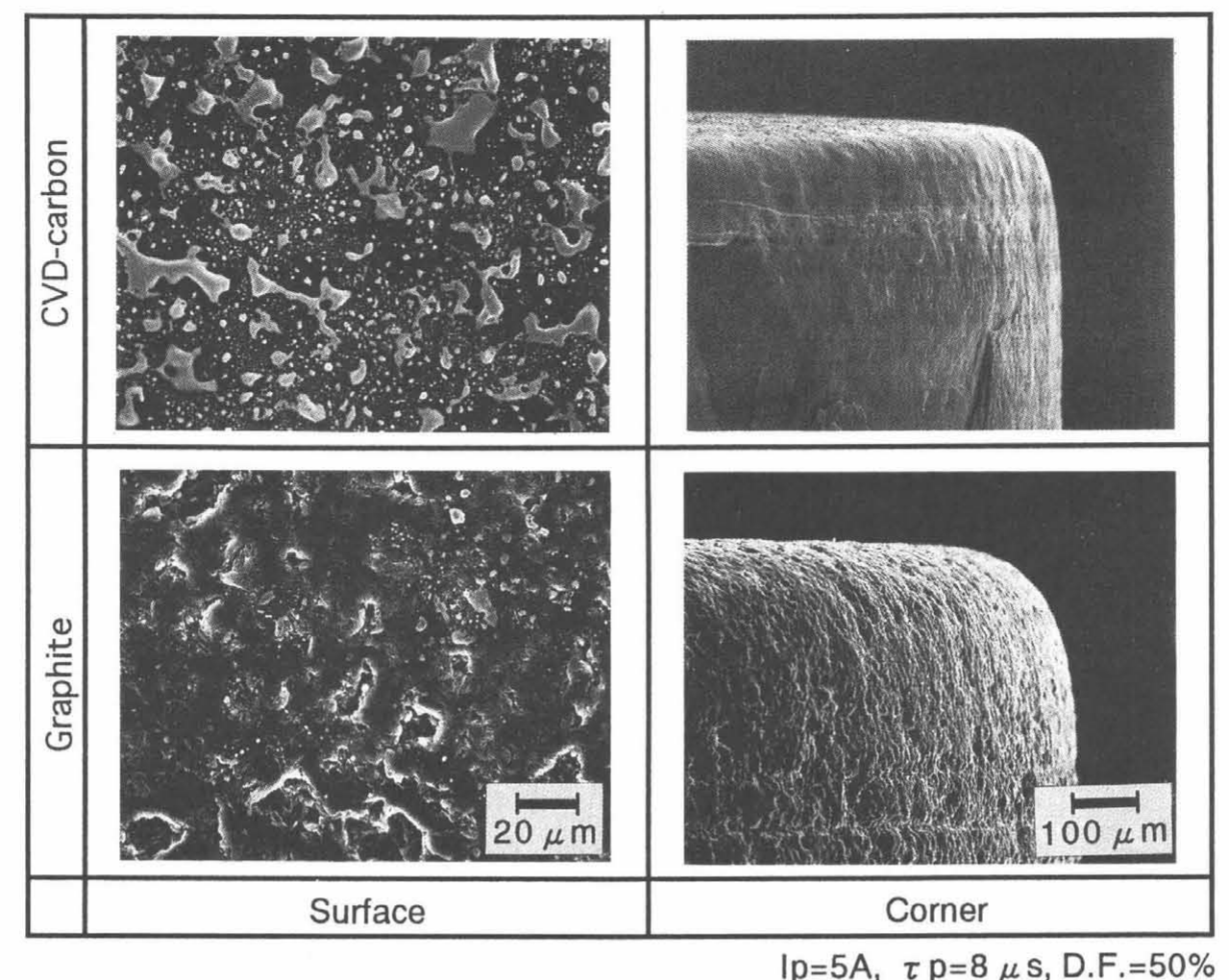

Fig.4 Micrographs of electrodes after EDM

both cases of graphite and copper. The electrode wear is more than $20 \%$ for the short pulse duration in the case of graphite electrode. On the other hand, it is very small in the case of CVD-carbon electrode. The value is about $1 \%$ even under the short pulse duration $\tau \mathrm{p}=4 \mu \mathrm{s}$. That is, EDM without electrode wear is almost possible in the finishing condition with CVD-carbon electrode. In EDM under the same condition with conventional electrode such as copper or graphite, EDM without electrode wear is impossible so far, though the improvement of the surface roughness is carried out. However, it is supposed from these results that this new CVDcarbon electrode has a possibility of overcoming the conventional limit. This is due to the high impact resistance because of its denser structure and high heat conduction in layer direction (about 4 times of graphite) as mentioned before.

Fig. 4 shows micrographs of end surface and corner part of electrodes after EDM under the finishing condition. As shown in the figure, adhesions of the workpiece material and the heat resolved carbon from the machining fluid are observed on the end surface of CVD-carbon, while there is no adhesion in the case of graphite. The adhesion of the heat resolved carbon is usually observed only under the long pulse duration and it leads to EDM with low electrode wear. The phenomenon shown in the case of CVD-carbon that the heat resolved carbon adheres to the electrode end surface even under the short pulse duration is very peculiar. Consequently, EDM with low electrode wear is attained under the finishing condition by using CVD-carbon electrode as can be seen in the previous figure. Then, on comparing the corner part where large electrode wear is usually observed, the corner in the case of CVD-carbon electrode relatively keeps its shape, while it wears and rounds in the case of graphite one ${ }^{\text {f. }}$. It is considered that the anisotropy of the resistivity and the heat conductivity improves the wear characteristics in the case of CVD-carbon.

Next, XPS (X-ray Photoelectron Spectroscopy) analysis of electrode end surface was carried out using S-Probe ESCA (Fisons Instruments, SSX100X). Fig.5 shows C1s photoelectron spectra of 
electrode end surfaces before and after EDM. The data contain the information from only $10 \mathrm{~nm}$ below the surface and the analysis area is $250 \times 1000 \mu \mathrm{m}$. The contamination layers such as oxidized layer and carbonized layer generated by the atmosphere was removed by argon ion etching in the measurement chamber before analysis. The dotted lines in the figure show the spectra before EDM. The binding energy of CVD-carbon is almost the same as that of graphite one, while the intensity of spectrum of the former is larger and the peak width is narrower than those of the latter. This is due to the uniform structure of CVD-carbon.

The solid lines show the spectra after EDM. The heat resolved carbon from the machining fluid adheres to both electrode end surfaces, since the pulse duration is long. That is, these are spectra of heat resolved carbon from the machining fluid during EDM. As can be seen in the figure, the binding energy of the heat resolved carbon adhering to the end surface of CVD-carbon electrode is larger than that of graphite one. The binding energy in XPS analysis represents the electrostatic energy between an atomic nucleus and electron. When the binding energy shifts to a larger energy (towards left) as shown in this figure, it means that the radius of the $1 \mathrm{~s}$ inner electron orbit decreases and the phase of chemical bond changes ${ }^{7}$. In other words, the structure of heat resolved carbon adhering to the end surface of CVD-carbon electrode differs from that of graphite one. Therefore, it is supposed that the former has a stronger wear resistance against heat and impact generated in electrical discharge than the latter. Consequently, the electrode wear with CVDcarbon electrode is very small as shown before.

Fig. 6 shows the relationship between the surface roughness and the pulse duration. As shown in the figure, the surface roughness increases with an increase of the pulse duration and takes a maximum at about $40 \mu \mathrm{s}$ in both cases. Under the short pulse condition, the surface roughness in the case of CVD-carbon electrode is smaller than that of graphite one. Considering this result together with characteristics of the electrode wear mentioned before, CVD-carbon electrode is very effective, especially under the finishing condition.

Fig. 7 shows variations of the removal rate with the pulse duration. The removal rate in EDM with CVD-carbon electrode is smaller than that with graphite one. In order to investigate the cause of this result, the EDMed surface was observed. Fig.8

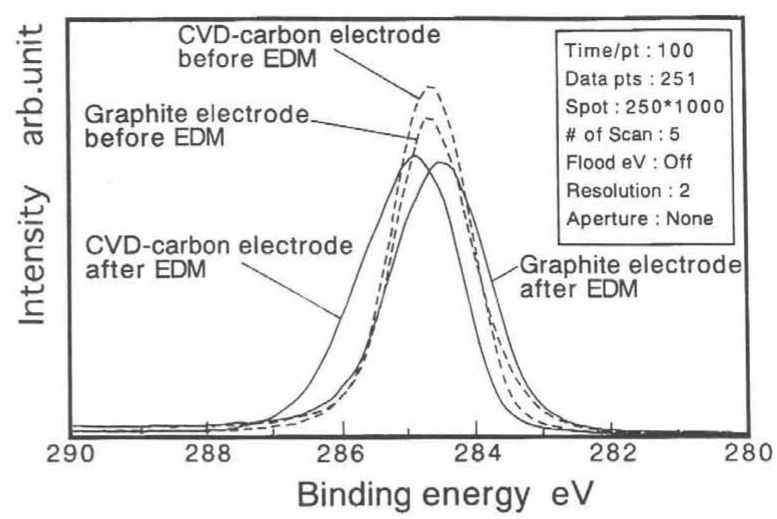

Fig.5 C1s photoelectron spectra of electrode end surface before and after EDM

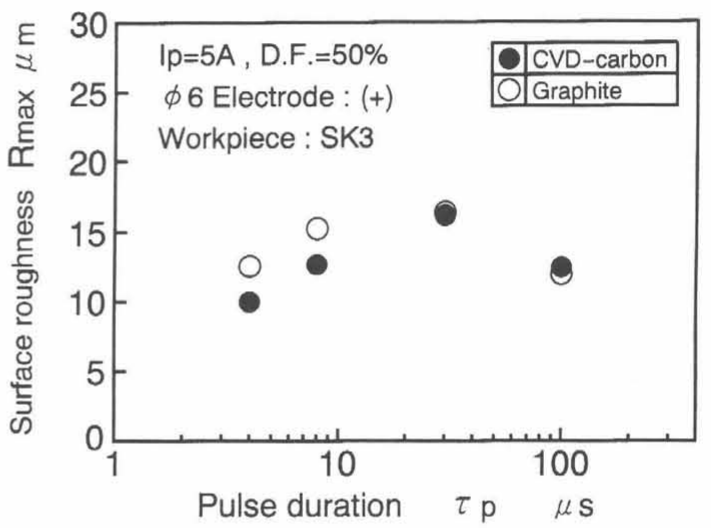

Fig.6 Relationship between surface roughness and pulse duration

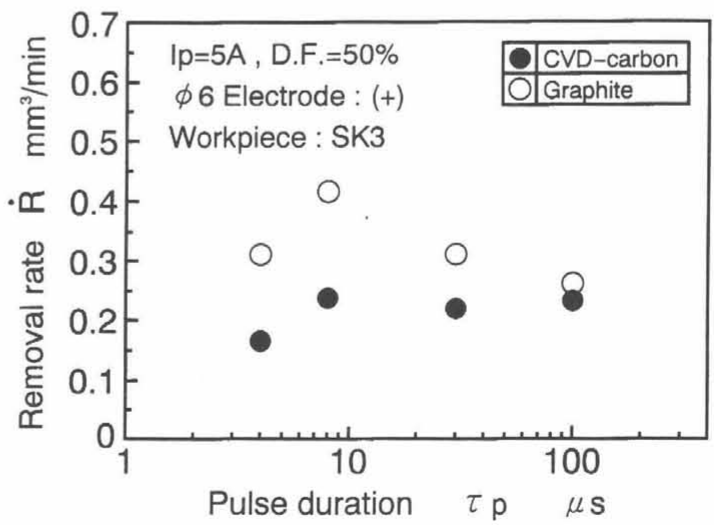

Fig.7 Relationship between removal rate and pulse duration 


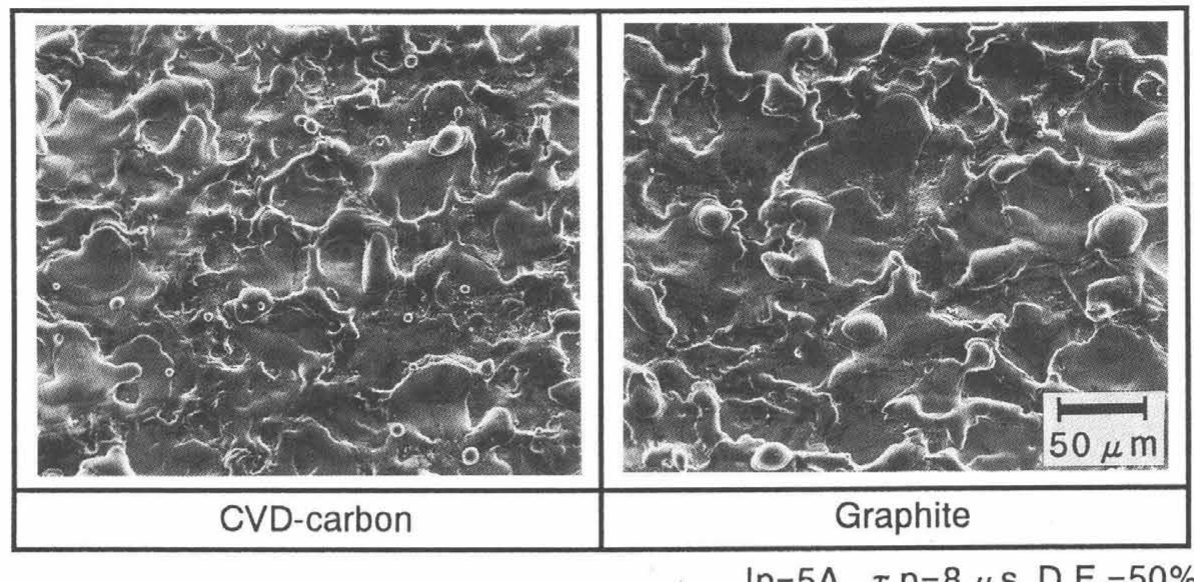

Ip $=5 \mathrm{~A}, \tau \mathrm{p}=8 \mu \mathrm{s}, \mathrm{D} . \mathrm{F} .=50 \%$

Fig.8 SEM micrograghs of EDMed surface

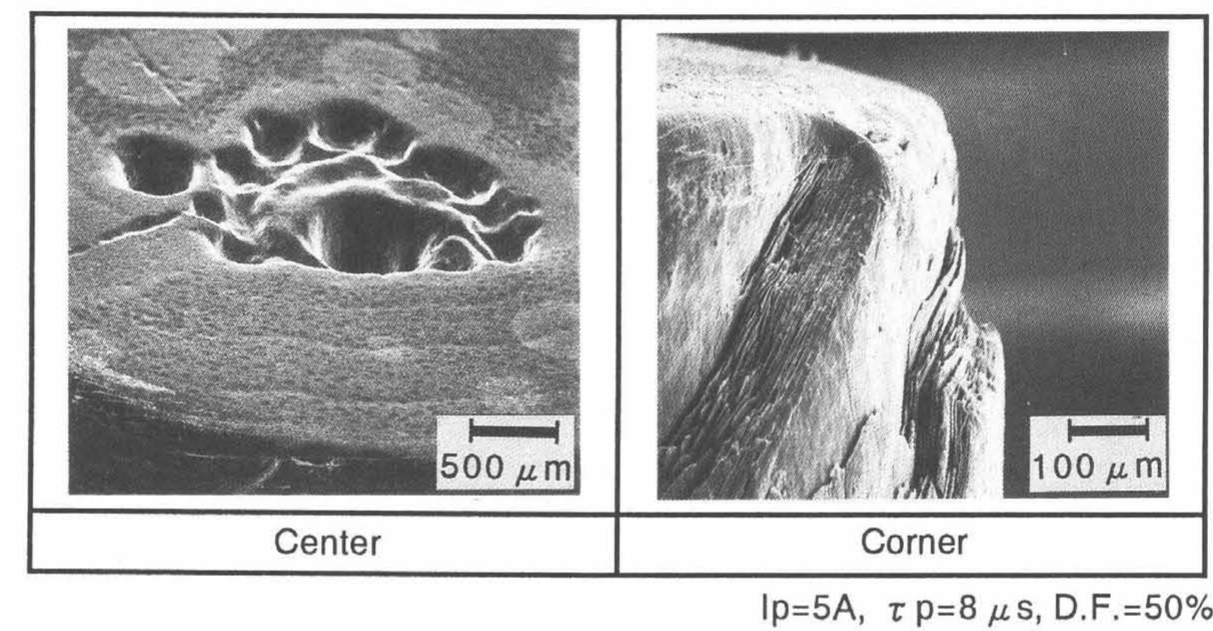

Fig.9 Center and corner parts of CVD-carbon electrode after EDM

shows SEM micrographs of machined surface. The size of crater on the machined surface with CVDcarbon electrode is smaller than that with graphite electrode. This is due to the difference of energy distributed to workpiece resulted from the kind of electrode materials ${ }^{8)}$. Therefore, the removal rate in the case of CVD-carbon electrode is lower and the surface roughness is smaller than those of graphite one as shown before.

From these results mentioned above, it is clear that CVD-carbon electrode has more excellent EDM characteristics compared with conventional one. However, some defects are observed as shown in Fig.9. In the center part, a deep depression was generated. In this part before EDM, carbon fibers existed as base material. It is considered that the cause is due to larger wear of carbon fiber than that of CVD-carbon. Moreover, CVD-carbon in some corner parts came off in layers during EDM. This is due to the frequent impact force generated in electrical discharge and the weak bond resulted from van der Waals' force in the deposition direction of CVD-carbon.

\subsection{Rectangular electrode}

It was made clear that EDM with low electrode wear rate under the finishing condition could be realized by using CVD-carbon electrode. However, the problem remains that the wear of base material part is relatively large as mentioned above. Then, a rectangular CVD-carbon electrode is made as shown in Fig.10 and evaluated. This electrode was divided 
from graphite base material plate and reformed after CVD-carbon was generated. Therefore, it does not include base material. Machining direction is the same as the layer direction as shown in the figure.

Fig.11 and Fig.12 show the electrode wear and the surface roughness respectively. The result of the same shaped graphite electrode is also shown. As shown in these figures, the electrode wear is very small and the surface roughness is a little small than those of graphite one under the short pulse duration, similarly in the case of cylindrical one.

\section{CONCLUSIONS}

EDM characteristics of a newly developed CVDcarbon electrode were experimentally investigated. Main conclusions obtained in this study are as follows:

(1)The heat resolved carbon from machining fluid has a stronger tendency of adhering to the end surface of CVD-carbon electrode than that of graphite one.

(2)EDM with low electrode wear rate is possible by using CVD-carbon electrode even under the finishing condition.

(3)At present, the newly developed CVD-carbon electrode has a defect of partly coming off of electrode, since the bonding force is low in the deposition direction.

\section{ACKNOWLEDGEMENTS}

The authors would like to thank Sodick Co., Ltd for their contribution of experimental apparatus.

\section{REFERENCES}

1) S.Nishimura and M.Tsuchiya : Study on Low Electrode Wear EDM, Journal of JSEME, 1, 2(1968)71. (in Japanese)

2) H.Xia, K.Kondo, M.Kunieda and N.Nishiwaki :Influence of carbon adhesion on electrode wear, Proc. of 3rd Annual Meeting of JSEME(1993)9. (in Japanese)

3) N.Saito : Principle of EDM and Its Application, GIJUTSU-HYORON-SHA(1979) 58.(in Japanese)

4) M.Suzuki, N.Mohri, N.Saito and H.Takezawa : A Study on the Electrode Wear in Electrical Discharge Machining, Journal of JSEME, 26, 52 (1992) 47.(in Japanese)

5)T.Ishikawa and T.Nagaoki : Recent Carbon Technology, KINDAI-HENSHU-SHA(1980)56.(in Japanese)

6) T.Yamashina and S.Fukuda : Fundamentals and Applications of Surface Analysis, TOKYO-DAIGAKUSHUPPAN-KAI(1991)116.(in Japanese)

7)Y.Kimoto : Precision Machining Applying Electrical and Electronic Principles, OHM-SHA(1982) 21.(in Japanese)

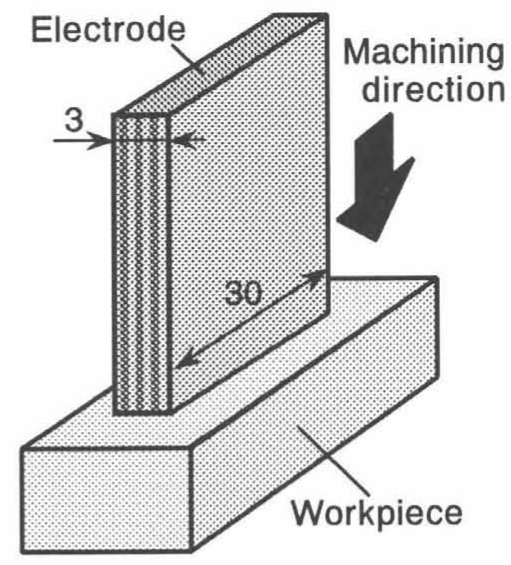

Fig.10 Rectangular electrode

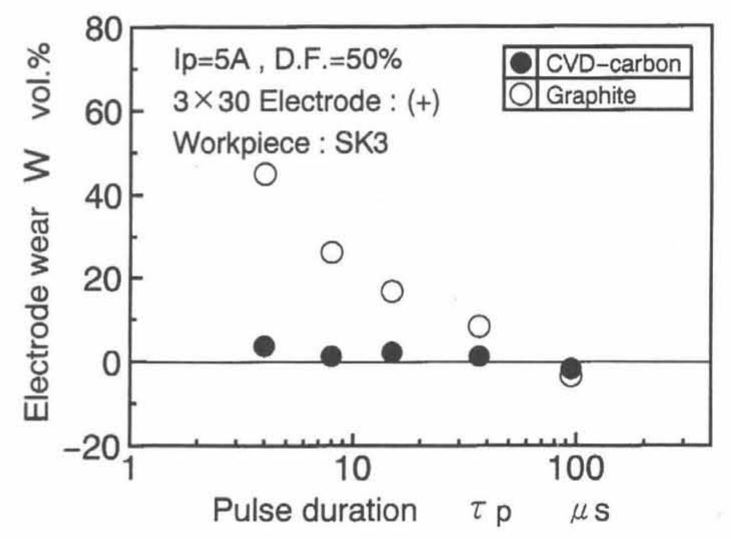

Fig.11 Electrode wear with rectangular electrode

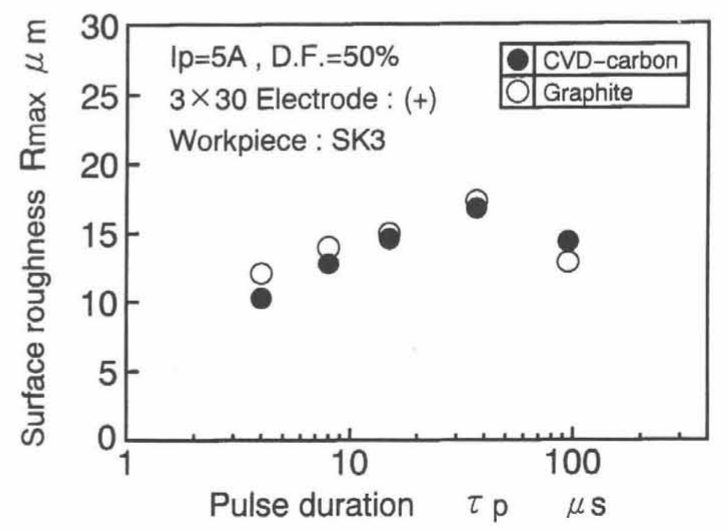

Fig.12 Surface roughness with rectangular electrode 